\section{VentriGel goes into pigs}

\section{By Kai-Jye Lou, Staff Writer}

Researchers at the University of California, San Diego and Ventrix Inc. have developed a catheter-compatible hydrogel that restored cardiac function in a pig model with myocardial infarction. ${ }^{1}$ Ventrix is now scaling up manufacture of the hydrogel, called VentriGel, and hopes to take it into clinical trials by year end.

In prior work, UCSD and Ventrix developed a hydrogel scaffold derived from porcine cardiac extracellular matrix (ECM) and showed that injecting it into rat hearts improved cardiac repair and function., ${ }^{2,3}$ The hydrogel was designed to mimic the mammalian heart's native ECM, which is important for proper cardiac function but is lost in the infarct region after a heart attack.

Now, the researchers have evaluated the same scaffold in a pig model of myocardial infarction (MI), which more accurately reflects human cardiac physiology.

In pigs, catheter-mediated delivery of VentriGel at two weeks post-MI increased cardiac function and decreased pathological left ventricular remodeling compared with delivery of saline control or no injection. Histology studies showed that VentriGel also increased cardiac muscle tissue and decreased fibrosis in the infarct region compared with controls.

A series of safety and biocompatibility studies showed that catheter-delivered VentriGel did not adversely affect peripheral tissues or cause arrhythmias in the pigs, nor did the hydrogel scaffold induce an immune rejection response or spontaneous formation of thromboemboli in rats. Finally, in human blood samples, the hydrogel did not affect coagulation.

Results were published in Science Translational Medicine. Ventrix cofounder Karen Christman, the corresponding author and an assistant professor of bioengineering at UCSD, led the research team.

"The current study is significant because it increases our confidence that our technology is working the way we think it is," said Ventrix CMO Paul Chamberlin. "The hydrogel scaffold improved wall motion in the heart, attenuated remodeling and didn't cause arrhythmias in the pig model. This work gives additional preclinical validation of our hypothesis that VentriGel, given post-MI, will help improve cardiac function."

Chamberlin added that VentriGel could be delivered to the heart using a minimally invasive catheter system, whereas most other injectable biomaterials being developed for cardiac repair need to be delivered via needle syringe, which would require an invasive surgical procedure.
"In many cases, other off-the-shelf injectable biomaterials are not capable of being delivered to the heart with a cardiac catheter," added Ventrix CEO and cofounder Adam Kinsey, a coauthor on the paper.

\section{Progressing into patients}

Ventrix's initial clinical plans are to develop the hydrogel scaffold in the EU. The company is scaling up GMP manufacturing of clinicalgrade VentriGel and expects to start a trial by year end.

Chamberlin said the company initially plans to evaluate cathetermediated delivery of VentriGel in patients with a recent MI event but thinks the product also could be applied to patients who are further removed from the MI event.

"Our early clinical plans will be to develop our product to treat patients in the acute MI scenario. Generally speaking, we want to initially target patients who have Class I or II heart failure, but we think the use of our product could eventually be expanded to the broader heart failure population as well," he said.

According to New York Heart Association guidelines, patients with Class I heart failure have cardiac disease but show no limitations in physical activity, whereas those with Class II heart failure show slight limitations such as fatigue, palpitations, dyspnea or angina resulting from normal physical activity.

Another possible scenario for VentriGel is in patients already undergoing surgery to restore cardiac function, said Harald Ott, a fellow in cardiothoracic surgery at the Massachusetts General Hospital and instructor in surgery at Harvard Medical School. "Patients undergoing a ventricular restoration procedure may achieve additional benefit from an injection of this hydrogel scaffold."

Moving forward, "it will be important to know how the ECM hydrogel treatment and revascularization either by percutaneous coronary intervention or bypass grafting could be used in conjunction with one another," said Jun Liao, assistant professor of biomedical engineering at Mississippi State University. "It would also be interesting to know how treatment with this ECM hydrogel would affect standard pharmacological therapies such as $\beta$-blockers and ACE [angiotensin-converting enzyme] inhibitors."

Ott suggested that the researchers may want to look into how other implantable, porcine-derived products were developed, as this could provide insights on how to further assess the biocompatibility and potential immunogenicity of the hydrogel scaffold.

Examples of such pig-derived products include the acellular porcine collagen tissue matrices CollaMend, Permacol, Strattice and XenMatrix, which are marketed for hernia and abdominal wall repair. The Davol Inc. unit of C.R. Bard Inc. markets CollaMend and XenMatrix. Covidien plc markets Permacol, and the LifeCell Corp. unit of Kinetic Concepts Inc. markets Strattice.

UCSD has filed for patents covering the technology described in Science Translational Medicine. The IP is licensed to Ventrix, and the company's VentriGel program is available for partnering.

Lou, K.-J. SciBX 6(10); doi:10.1038/scibx.2013.232

Published online March 14, 2013 


\section{ANALYSIS}

\section{REFERENCES}

1. Seif-Naraghi, S.B. et al. Sci. Transl. Med.; published online Feb. 20, 2013; doi:10.1126/scitransImed.3005503

Contact: Karen L. Christman, University of California, San Diego, La Jolla, Calif.

e-mail: christman@eng.ucsd.edu

2. Singelyn, J.M. et al. Biomaterials 30, 5409-5416 (2009)

3. Singelyn, J.M. et al. J. Am. Coll. Cardiol. 59, 751-763 (2012)
COMPANIES AND INSTITUTIONS MENTIONED

Covidien plc (NYSE:COV), Dublin, Ireland

C.R. Bard Inc. (NYSE:BCR), Murray Hill, N.J.

Harvard Medical School, Boston, Mass.

Kinetic Concepts Inc. (NYSE:KCl), San Antonio, Texas

Massachusetts General Hospital, Boston, Mass.

Mississippi State University, Mississippi State, Miss.

University of California, San Diego, La Jolla, Calif.

Ventrix Inc., San Diego, Calif. 